\title{
Surgery of IC-Anterior Choroidal Aneurysms
}

\author{
Thomas Kretschmer, Christian Heinen, Julius July, \\ and Thomas Schmidt
}

\subsection{Signs and Symptoms}

Anterior choroidal artery aneurysms (AChAA) are comparatively rare and appear with a frequency from around 2-4\% to $10 \%$ in larger series, e.g., M Lawton personal series of 2500 clipped aneurysms: $n=98 \mathrm{AChA}=4 \%$ [1]. Number of AChAA in treated aneurysms group of BRAT study $2.2 \%$, median aneurysm size $7 \mathrm{~mm}$, total 9 cases of 408 treated; 209 were clipped and 199 were coiled [2]. Kuopio aneurysm data base from 1977-2005, consist of 3005 patients with 4253 aneurysms, there were 99 (10\%) AChAA in 70 (8\%) patients [3].

There are no characteristic clinical findings for unruptured AChAA. Not infrequently they are detected during work-up of other ruptured or non-ruptured aneurysms. AChAA already can rupture at small size due to their characteristics of thin wall and orientation aligned along the main blood jet from the ICA. Rupture of AChAA can lead to blood distribution favoring the ipsilateral side and basal cisterns, comparable to PCoA aneurysm rupture.

T. Kretschmer $(\bowtie) \cdot$ C. Heinen · T. Schmidt Department of Neurosurgery, Evangelisches Krankenhaus Oldenburg, Oldenburg, Germany

J. July

Department of Neurosurgery, Faculty of Medicine Universitas Pelita Harapan (UPH), Neuroscience Centre Siloam Hospital Lippo Village,

Tangerang, Indonesia
Ruptured AChAA usually will show blood at lateral suprasellar and ambient cisterns [3]. Very rare it presents with subdural or intracerebral blood collections despite proximity of the aneurysm to the temporal lobe. Intraventricular breakthrough of hemorrhage will be more prominent in temporal horn. Cranial nerve deficits seem to be infrequent; however oculomotor insufficiency has been described in ruptured or unruptured cases [4].

There are specific aspects about the AChA aneurysms especially the anatomy and its variant.

\subsubsection{Anatomy}

The AChA is a phylogenetically "old," respectively, early vessel, which is prominently developed until the 5th week during human embryology (choroidal stadium). The anterior circulation supplies almost the whole brain during this phase. Over hemispheric growth additional supply by the vertebrobasilar system is necessary. As a consequence the brain volume supplied by the AChA is progressively reduced. The AChA may show various anatomical variations.

The AChA originates 2-4 mm distal of PCoA on the posterolateral part of ICA, almost always, lateral to optic tract. Its diameter is only about $1 \mathrm{~mm}(0.5-1.5 \mathrm{~mm})$. The artery then runs below the optic tract from lateral to medial. It courses 
along the perimesencephalic cistern (cisternal segment), entering temporal horn of lateral ventricle at choroidal fissure ("plexal point"). Within ventricle (choroidal segment), it runs along the choroid plexus to anastomose with branches of the lateral and medial posterior choroidal artery (PChA).

Despite its relative small size and short length, it supplies some major territories: AChA perforators supply the optic tract, optic radiation, crus cerebri, hippocampus, corpus amygdaloideum, substantia nigra, globus pallidus, lateral thalamus, and the posterior crus of the internal capsule. Along its course AChA may send off as many as 16-20 separate branches.

In proximity to the $\mathrm{AChA}$ origin, there may be one or several perforator branches to the temporal lobe. The optic tract gets blood supply from perforator of anterior choroidal artery that passes medially and turns around the optic tract and then penetrates the tract superiorly. There are also variants where perforators go through the optic tract and supply internal capsule and globus pallidus. Other perforators pierce peduncle and supply red nucleus and substantia nigra. Following the peduncle, the lateral geniculate and the pulvinar are crossed before entering the lateral ventricle choroid plexus.

\subsubsection{Variants}

AChA may be duplicated in $4 \%$ of cases. Ectopic ramification with AChA originating from posterior cerebral artery (PCA) or middle cerebral artery (MCA) has been described. Origins of AChA and PCoA usually are 3-4 mm apart and, however, on occasion may be simultaneous or only 1-2 mm apart. Very rarely the AChA comes off the ICA proximal of the PCoA origin. Rare variants, whereby AChA supplies the occipital lobe, including the calcarine cortex, were described.

\subsection{Preoperative Investigation}

AChAA may be hard to differentiate from PCoA aneurysms on angiograms. In comparison to PCoA aneurysms, they project in a more lateral direction and may point into the uncus of the temporal lobe. It is crucial to identify the AChA as such and to determine whether it arises as a single trunk or as multiple branches.

$\mathrm{CT}$ and thin slice CT angiography with $3 \mathrm{D}$ reconstruction will raise suspicion for an AChAA; however it does not suffice to clearly depict details of vessel-to-aneurysm topography and exact site and course of emanating branches. As such, contrary to some of the other aneurysm locations, we feel catheter angiography is precondition to further treatment decision. Depending on the result of imaging studies, branching pattern, age, and condition of the patient, we decide whether to clip or coil. Angioarchitecture at the aneurysm neck plays an important role in that decision, with branching directly at the neck favoring aneurysm clipping and clip reconstruction of the AChA.

$3 \mathrm{D}$ rotational angiography is a very valuable tool to dismantle the true topography. Frequently the aneurysm will project laterally and straight to caudal with respect to the ICA. So AP and lateral views are very important to resolve the aneurysm sac projection. The AChA itself will best be seen in a lateral and sometimes basal view and needs to be discerned from the PCoA (Fig. 12.1). From its origin on the lateral ICA aspect, the artery courses posteriorly for 4-5 mm and short medially to optic tract to follow its course laterally around the peduncle. This curvature can easily be detected on a lateral view. The differentiation is more difficult on an AP view: here the AChA describes a medial curve, to then turn laterally and upward, to make a sudden kink at the plexal point.

\subsection{Presurgical Preparation}

We do prepare as for any other aneurysm clipping. One exemption being an additional reciprocating saw (with very slender blade) and chisels in the rare occasion, we plan to place an orbital extension of the standard lateral supraorbital approach (LSO). Patients will have a CV line (central venous line); we do not routinely order blood products in unruptured cases. After patient 

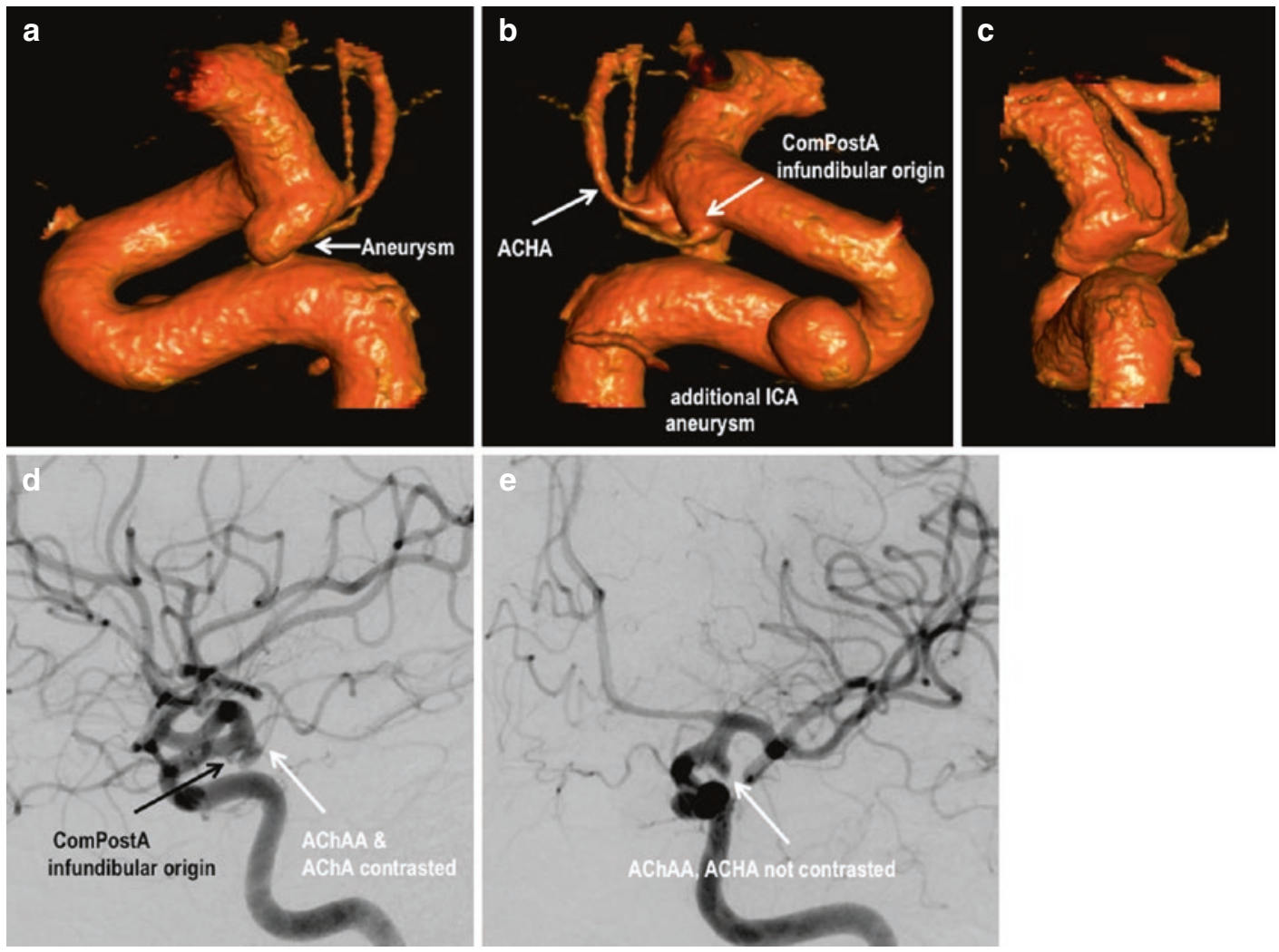

Fig. 12.1 (a-e) Preoperative imaging. Angiographic depiction of an AChAA from different views including 3D reconstruction (upper row). (a) Lateral view on aneurysm with AChA emanating from dorsal neck. (b) Medial view of AChAA neck, with infundibular PCoA close to aneurysm. Extradural aneurysm of ICA, as secondary finding. (c) Dorsal view on aneurysm and emanating AChA. PCoA and ACA

(A1) can also be depicted. (d) Lateral view of angiographic image, infundibular PCoA branches off close to AChA, as well as the more distal (flow direction) broad-based AChAA neck and AChA emanating from there. (e) Slightly oblique $A P$ view. With this alignment only the aneurysm is perceptible, however, not the branching AChA. To depict the AChA in this case, images turned further are necessary

and head clamp positioning, the patient is prepped and draped. During this procedure, he will receive $1 \mathrm{~g} / \mathrm{KG}$ of mannitol if not the kidney is not compromised. Standard trays for craniotomy and vascular microsurgery are prepared. We have a standard clip sieve on the table, which is assembled to our needs. It contains the clip forms and sizes we use most of the time; only "exotic" clips will be directly unpacked as needed. A straight aneurysm clip and two types of temporary clips (7 $\mathrm{mm}$ bent and straight) are taken out of the sieve and placed ready on the instrumentation table for cases of premature rupture. Adenosine (for induction of temporary cardiac standstill) as well as indocyanine green (ICG) will be available

on the anesthesiology side before we start. Anesthesiology will be prepared to enable temporary clipping by bolus administration of thiopental with $100 \%$ inspiratory oxygen concentration at any time during the procedure. Imaging studies are depicted; monitors for anesthesiology and OR care are available to follow the procedure.

\subsubsection{Approach}

The initial microsurgical strategy is similar to those of PCoA aneurysms, as these arteries emanate in close vicinity. However, as the AChA and 


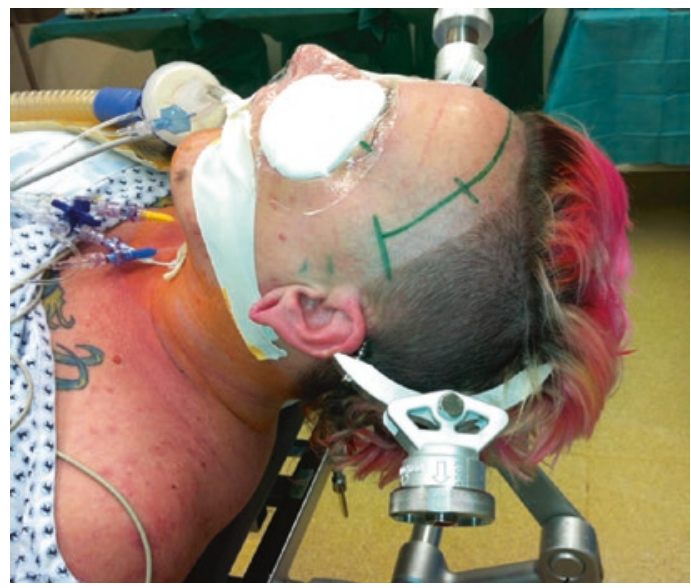

Fig. 12.2 Positioning. Head elevated well above heart level, slightly reclined toward the floor, and only minimally turned toward the right shoulder (about $20^{\circ}$ ). Planned incision for lateral supraorbital approach (LSO) with orbital deroofing. For LSO incision is at hairline margin, starting $2-3 \mathrm{~cm}$ above zygoma (if such a frontally placed incision is chosen, it needs to end well above the zygoma; otherwise the facial nerve is jeopardized); length of incision depends on the approach chosen and hairline distance from eyebrow. For simple LSO 6-8 cm suffice

its perforator takeoff are hidden by aneurysm sack and ICA, the specific anatomy with high likelihood of perforators directly emanating at the aneurysm neck makes its treatment more challenging. Standard approaches such as pterional, lateral supraorbital (LSO), and supraorbital keyhole are used most frequently. Our personal choice is standard LSO [5]. In cases of expected obtunded view on AChA origin, we might choose an orbital extension of the LSO in the form of a combined one-piece orbital rim deroofing to gain some extra degrees of surgical dissection corridor (cranio-orbital approach).

Patient is placed supine, and the head is higher than heart level, moderately reclined, and minimally turned to the contralateral side by about $20^{\circ}$ (Fig. 12.2). If the head is turned too much, the temporal lobe is rotated over the aneurysm in the visual trajectory of the surgeon. Correct head position avoids the need for a fixed retraction device in particular for temporal lobe retraction.

The incision is placed at the hairline and begins $2.5-3 \mathrm{~cm}$ above the zygoma. Six to seven centimeters of length usually suffice if galeal flap is well retracted by spring or rubber-connected fishhooks (Fig. 12.3). It is important to expose zygomatic process of frontal bone in order to enable a craniotomy cut down to the fronto-sphenoidal base.

For the standard LSO, we place one burr hole below superior temporal line, $2-2.5 \mathrm{~cm}$ posterior to the zygomatic process, as we do not see the need for the so-called keyhole burr hole. A minicraniotomy is fashioned without additional burr hole cut very precisely to the cranial base. The inner bony rim (internal tabule) and cranial base are flattened out to enable unimpeded vision with a drill or the unguarded craniotomy burr.

\subsection{Surgical Steps}

Following craniotomy, dura is incised to create a frontally based flap that is pulled over the frontal base and fixed with dural stitches. Low-profile craniotomy and low dural opening enable for a comfortable subfrontal dissection corridor. After introducing the operating microscope, a first subfrontal dissection trajectory starting from the frontal "zygomatic process pillar" in a perpendicular direction will directly lead to the ipsilateral optic nerve and optic cistern (Fig. 12.4a).

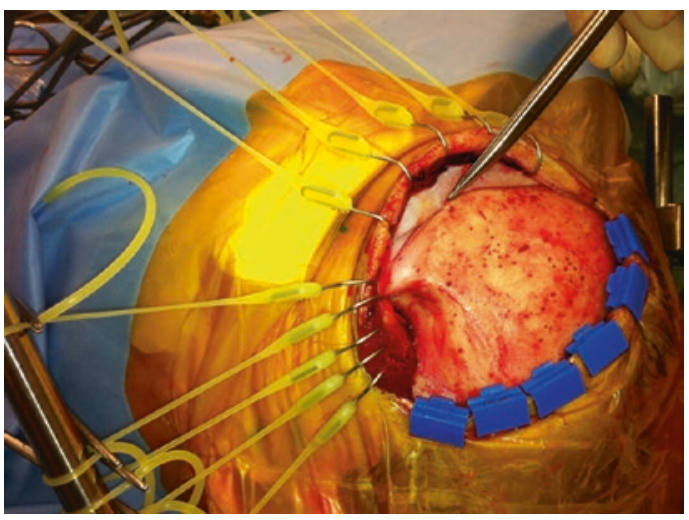

Fig. 12.3 Incision and exposure. Exposure after a $7 \mathrm{~cm}$ skin incision and having raised a combined galeal-muscle flap by incising through fascia and muscle. Muscle is carefully stripped from bone to prevent later atrophy. In this case orbital rim was fully exposed for cranio-orbital approach. In routine LSO it is not necessary to detach that far down; to expose beginning of zygomatic process of os frontale is enough to cut craniotomy flush with the frontal base 

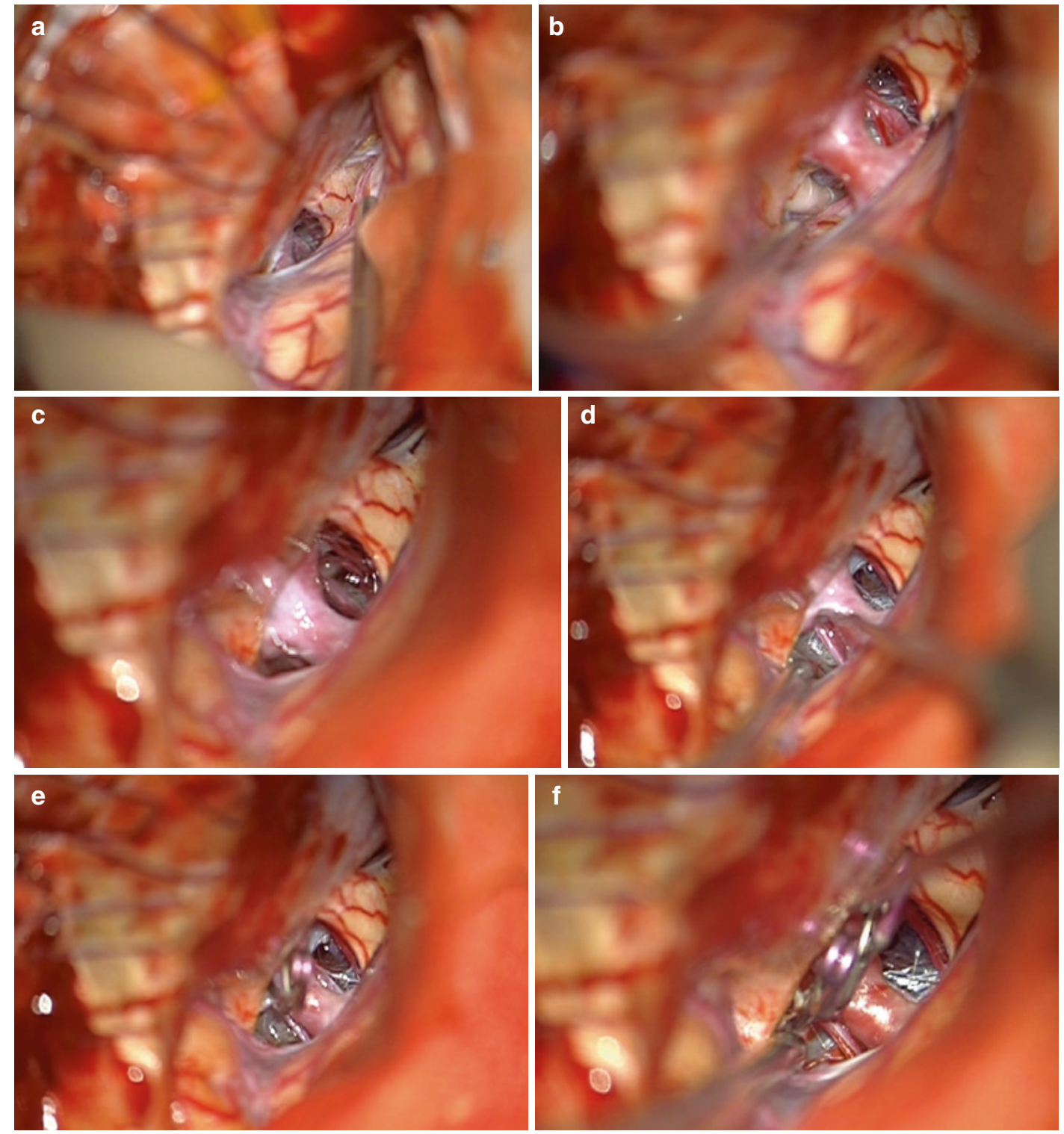

Fig. 12.4 (a-f) Aneurysm clipping. (a) Beginning of intradural part. Subfrontal exposure and opening of optic cistern. (b) Distal ICA after head has been lowered depicts bifurcation and A1. (c) Distal part of aneurysm neck. ICA hides AChA. (d) After deflecting the ICA with dissector,

The optic cistern, medial to optic nerve, is safer to be opened first, as compared to the opticocarotid cistern on the lateral side in ruptured cases. CSF drainage via the cistern will further relax the brain and progressively open a subfrontal corridor by using gravity without any fixed retraction. After opening the optico-carotid cis-

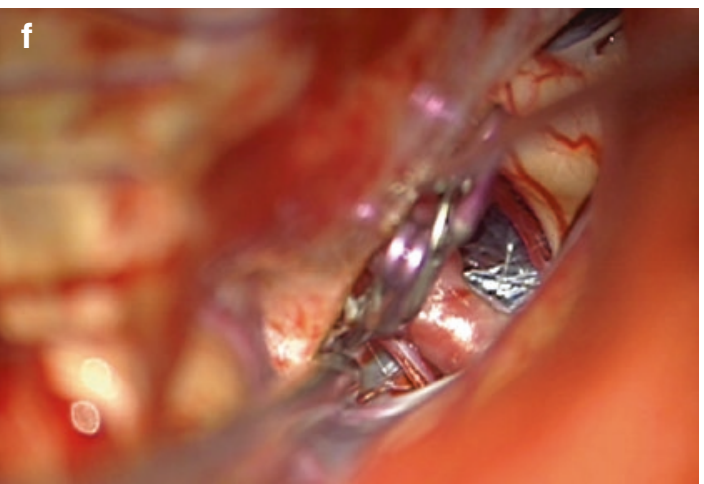

AChA can be seen. (e) As neck area and AChA have been identified, safe clipping is now possible. (f) Second smaller clip is needed for neck area to reconstruct beginning of AChA wall

tern, subfrontal adhesions are progressively loosened. Although the ICA is the target and will readily be in the operative view, additional freeing of the temporal and frontal lobe, allowing them to fall back, opens the available dissection space. However, microsurgical dissection should begin without any temporal lobe retraction to 
avoid premature aneurysm rupture by opening first the optic and then the optico-carotid cistern to gain a surgical corridor sufficient for ACI dissection. The superomedial surface of the ICA is safe for dissection. Once the proximal ICA is freed and centered in the obtained view, additional room can then be gained by splitting carefully just the beginning of the proximal Sylvian fissure overlying the carotid T.

After the more distal ICA is freed from arachnoid adhesions, the PCoA and the oculomotor nerve can be identified (Fig. 12.4b). The aneurysm should be located distally to the PCoA origin (Fig. 12.4c). Once the AChA is identified, it needs to be determined if the AChA arises as a single trunk or multiple vessels (Fig. 12.4d). AChAA may arise from a proximal or distal segment. Large or giant AChAA (quite rare) might be adherent to the uncus of the temporal lobe or rarely to the oculomotor nerve. Frequently the aneurysm sac or the ICA itself (Fig. 12.4d) will hide the AChA that is emanating behind the sac. Safe clip application necessitates maneuvers that ascertain direct view on the ICA at the aneurysm neck and AChA takeoff. Sometimes this might only be possible by use of a transient dynamic maneuver displacing the main trunk with a microdissector. This might encompass active compression or displacement of the aneurysm dome with a microdissector to gain sight to potentially hidden perforators (only in unruptured aneurysms). All these maneuvers are potentially dangerous as they do bear a high risk of premature rupture but might be the only means to directly assess the "takeoff area." Besides experiencing their use necessitates heightened awareness for premature rupture and knowledge of "bailout" strategies, e.g., temporary clipping, cardiac standstill, handling of ICA lacerations, use of fenestrated clips, and vessel wall reconstruction techniques with clips. Measures to gain view and change trajectory also include turning of the OR table in the longitudinal axis of the patient and lowering of the OR table head/backpiece. Escalated measures are short cardiac standstill by adenosine (one dose will give 20-40 s), sometimes even temporary ICA clip- ping (only very short tolerance time, if possible needs to be avoided by full use of other alternatives).

After optimal aneurysm exposure, preparation for clip application is undertaken. Temporary and permanent clips should be readily available. A potential site for temporary clip application and choice of the temporary clip is chosen. For clipping the shortest clip amenable should be chosen. In uncomplicated aneurysms a straight or slightly laterally bent clip might be appropriate. Complex aneurysm architecture at the neck might necessitate clip combinations to reconstruct the AChA and/or perforator takeoff (Fig. 12.4e, f). At times it is warranted to place a pilot clip to secure the dome first, before the neck is reconstructed. Small branches adherent to the aneurysm wall not amenable to dissection might be excludable from clipping them off by use of a tandem technique with a fenestrated and a straight clip.

If a complex branching pattern or obtunded view can be foreseen, we are inclined to use a one-piece mini-cranio-orbital extension of the standard LSO from the start of the craniotomy (Fig. 12.5). This will add several degrees of dissection corridor (from a caudal to cranial direction in the sagittal plane) and allows to lower the headpiece of the OR table further, as the orbital rim is not in the field of view. However, an optimal view on the branching site cannot always be achieved.

Adjuncts to delineate the neck and perforator situation after clipping might be an angled endoscope (however we mostly find it too bulky in that location), ultrasound mini-Doppler, and ICG. To avoid misinterpretation of results, it is advisable to test already before clipping.

After clipping and ICG/ultrasound control of perfusion, we fill the operative site with Ringer's and close dura. The wound is closed meticulously in layers. We separate adaption of the muscle and fascia as we do prevent drains. Also in a minicranio-orbital approach in small incisions (ca. $7 \mathrm{~cm}$ ), prevention of swelling, hematoma, and pristine, unchanged cosmetic are obtainable with careful handling of soft tissue and bone (Fig. 12.6a, b). 

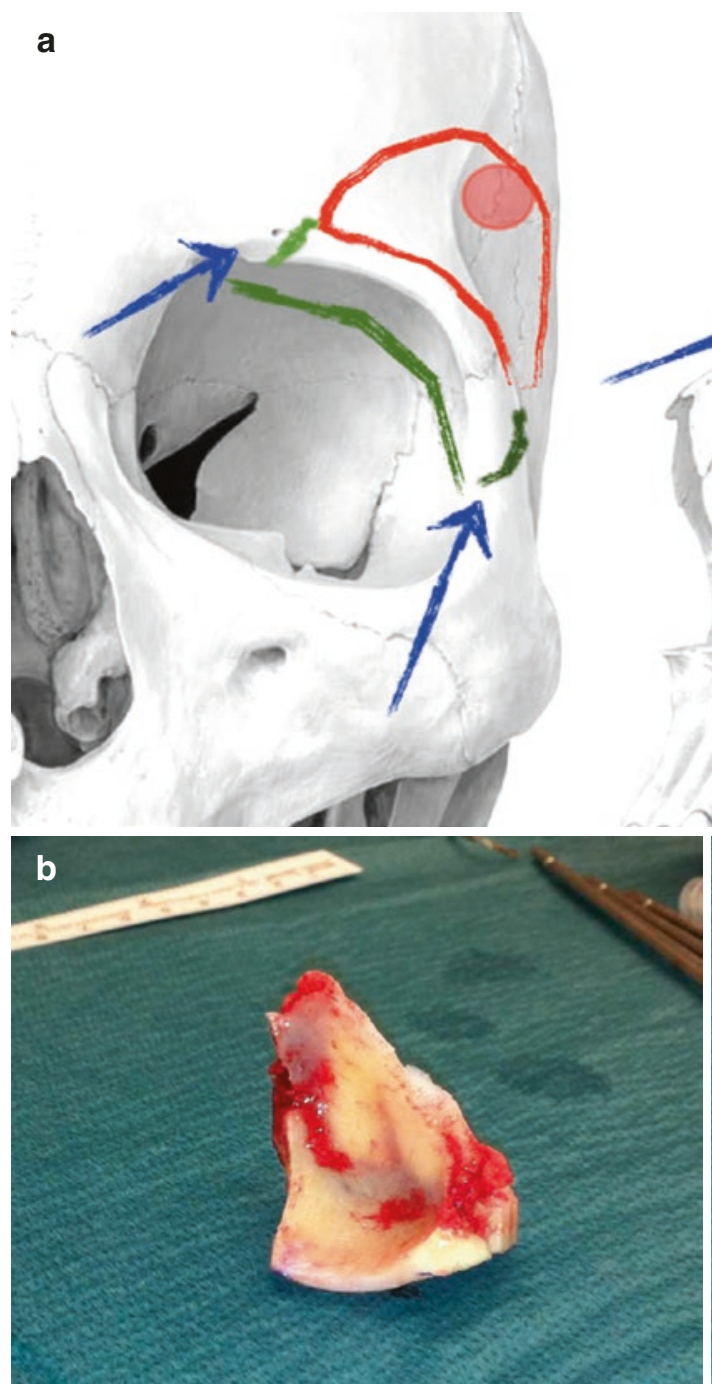

Fig. 12.5 (a-c) Craniotomy. Usually a standard LSO (red line) will suffice. In this we prefer to place one burr hole below superior temporal line and then cut a craniotomy down to the frontal base and across the sphenoid bone. In the depicted case, however, a one-piece minicranio-orbital approach was chosen with orbital deroofing (green line, instead of red frontal base line). (b) Shows inner side of the orbital roof. We prefer to incise medial and lateral orbital roof with very thin reciprocating saw (blue arrows in a) to have a sharp cut with minimal bone loss and split the rest with small chisels that will create a triangular shape (green line in a). (c) Shows the outer side with burr hole, corresponding to (a) right side 

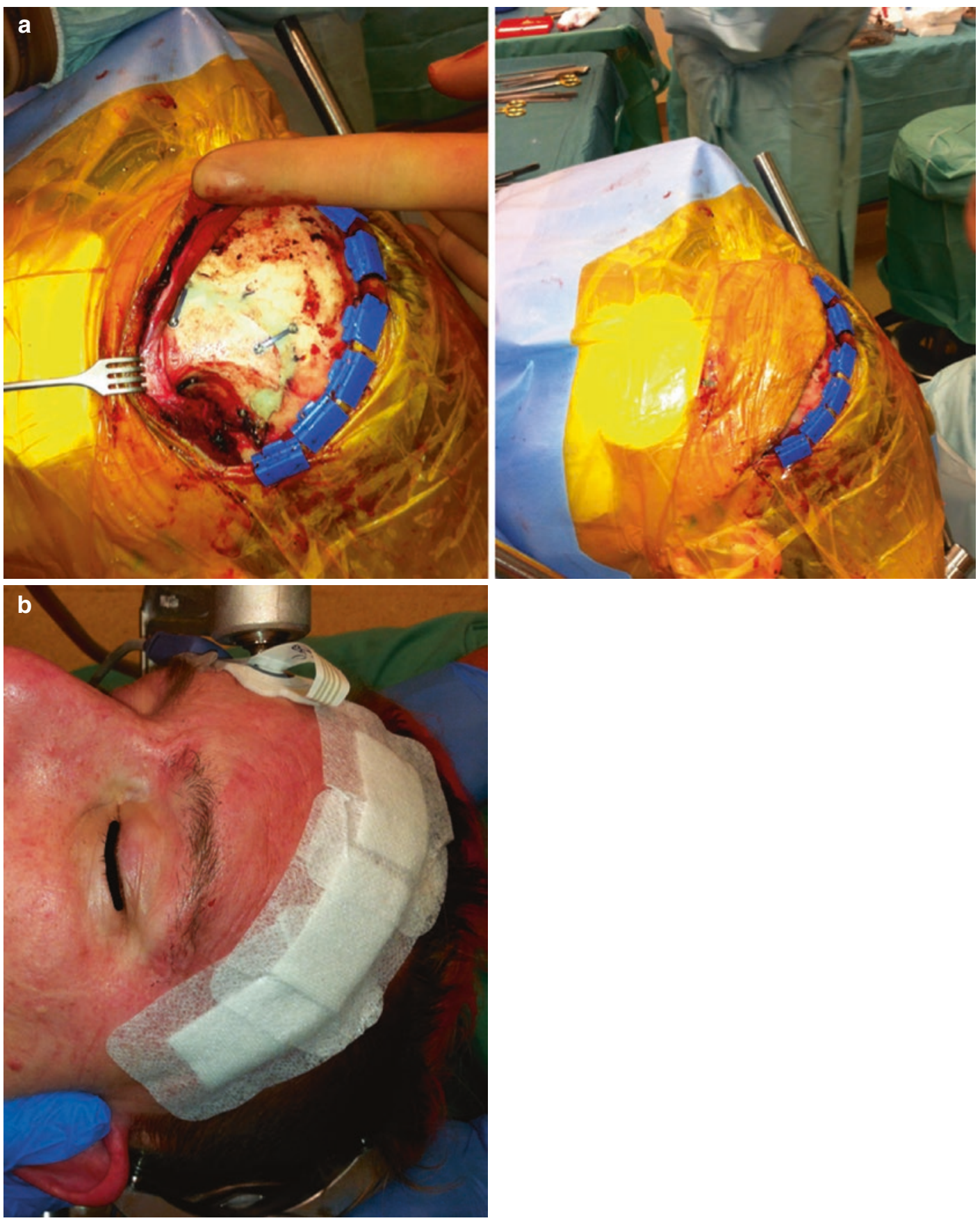

Fig. 12.6 (a, b) Closure. (a) Bone flap is repositioned and secured in place with mini-plates. Craniotomy groove and burr hole have been sealed with bone cement, followed by meticulous suture in layers (muscle and fascia

separately, galea, skin). We do not routinely use drains. (b) Demonstrates cosmetic result with absence of orbital hematoma or swelling 


\subsection{Complication Management and Avoidance}

Treatment of AChAA bears a far higher risk for complications. The main risk is inadvertent occlusion of the AChA with the devastating consequences of hemiparesis to hemiplegia and hemianopia. This applies to surgical clipping and interventional coiling alike. Premature rupture is another severe risk. Rupture is more difficult to handle as compared to other aneurysm locations.

Meticulous dissection, optimization of view trajectory by combination of adequate exposure around ICA, and patient positioning via table tilt are necessary to preset for optimal clipping conditions. Provisions for temporary clipping/cardiac arrest should have been made in advance, before skin incision. Median nerve SEP monitoring is a valuable tool to detect early signs of ischemia, in case the AChA is compromised during the course.

Indocyanine green (ICG) angiography, ultrasound (US) mini-Doppler to assess outflow, and papaverine/nimodipine irrigation after temporary clipping are adjuncts to assess vessel patency or treat spasm.

Premature aneurysm rupture can be controlled with a pilot clip across the aneurysm sac. This should then give enough time to assess the local topography in order to reapply the clip or place an additional one. If this is not feasible, placement of a pilot clip under pharmacological adenosine cardiac arrest is another option.

In case of devastating rupture of ICA/aneurysm neck, which cannot be handled by suction alone within the above-stated time constraints, we recommend to assess the tear by temporary clipping of ICA (preferably within $30 \mathrm{~s}$ to $2 \mathrm{~min}$ ). If the ICA lumen cannot be clip reconstructed, a small circular cotton wrap around ICA is placed and secured in place with an angulated aneurysm clip. If bleeding does not cease after the circular cotton sheath in place, additional absorbable fibrin sealant patch $\left(\right.$ TachoSil $\left.^{\circledR}\right)$ is added. Small rents can be sealed with tiny TachoSil ${ }^{\circledR}$ pledgets and cottonoid in a first attempt. All in all it will be very difficult to save the AChA in such a circumstance.

In cases of inadvertent closure of AChA by embolus, emergent bypass or open embolectomy is not really feasible at this location and thus do not represent realistic bailout options.

The incidence of procedure related to permanent AChA syndrome after surgical clipping of an AChAA ranges from 5 to $16 \%[4,6]$. AChA syndrome manifests with contralateral hemiparesis to hemiplegia and more inconsistently with contralateral hemianesthesia or hemianopia.

\subsection{Postoperative Care and Follow-Up}

We do not use drains on a routine basis. The wound is closed meticulously in layers to prevent blood accumulation (temporalis muscle and fascia are closed separately). Skin is closed with staples or suture that will be removed after 7 days.

ICG angiography is used on a routine basis intraoperatively; however it has no power to reliably rule out residual perfusion. Regardless of intraoperative evaluation, we do perform postoperative catheter angiography to confirm complete clipping in every aneurysm case regardless of location (Fig. 12.7). In our own practice, this will be done in the direct postoperative period. In non-ruptured cases catheter angiography will be performed within the next 2 days to enable early discharge of the patient.

The patient is discharged within 3-7 days after surgery in non-ruptured cases. Length of stay in subarachnoid hemorrhage (SAH) patients is dictated by the course of the disease, length of intensive care treatment, overcoming of the spasmic phase, sequelae of hemorrhage, and also availability of a rehabilitation placement.

The first follow-up on our clipped patients will be 3 months after discharge at our outpatient clinic. 

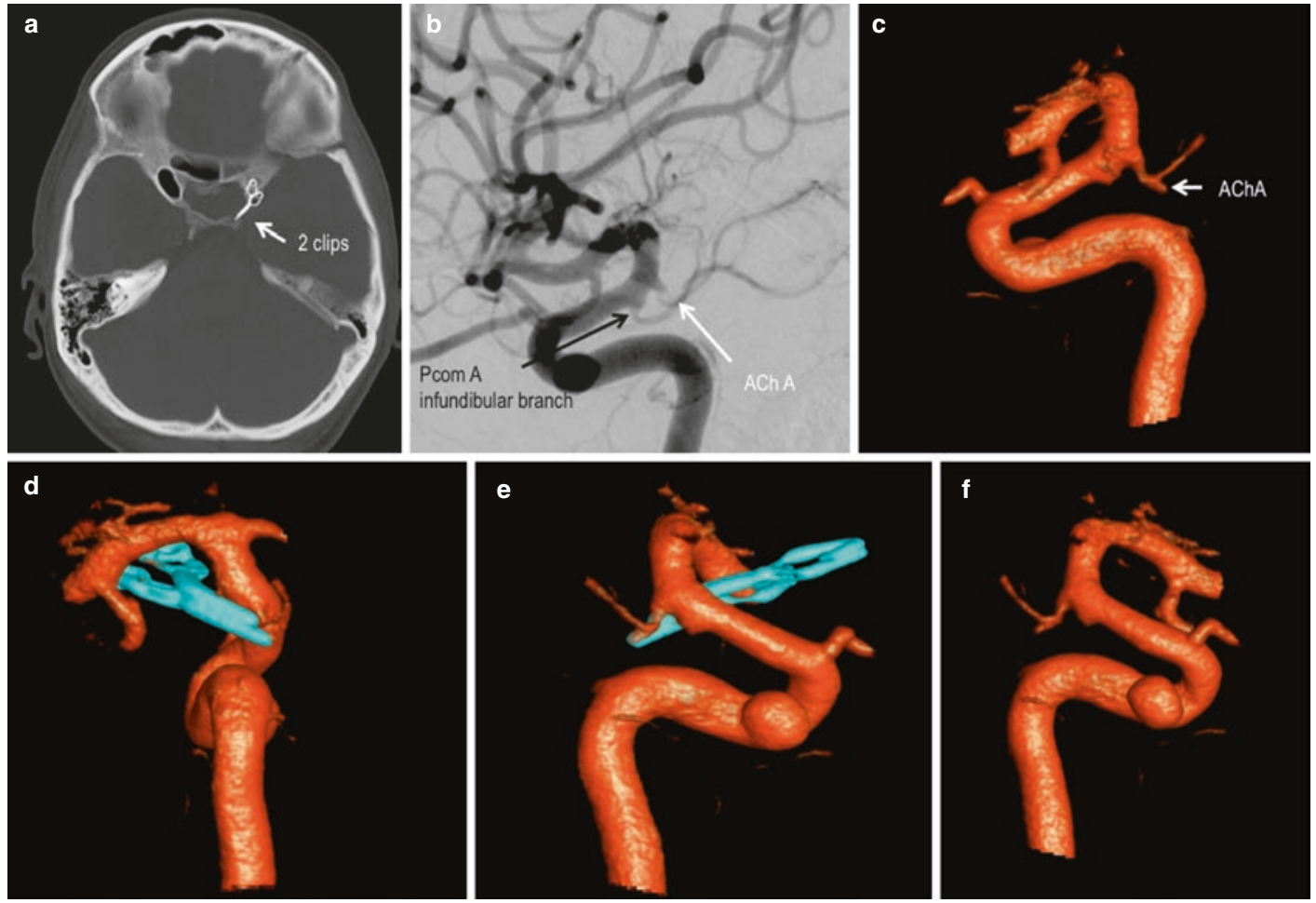

Fig. 12.7 (a-f) Postop imaging. Clip position in relation to bony skull base on $\mathrm{CT}$, postop angio, and 3D rotational angio reconstructions with and without clips. (a) Basal view on placed clips in CT. (b) Lateral view of ICA with

\subsubsection{Pearls}

AChAA are rare but bear a higher risk of rupture and are more difficult to treat. They are frequently thin walled and have the main AChA branch in direct vicinity to or emanating from the aneurysm neck. As such the risk of ischemic complications due to surgery is comparatively high.

\section{References}

1. Lawton MT. Seven AVMs. Thieme;2014.

2. Spetzler RF, McDougall CG, Albuquerque FC, Zabramski JM, Hills NK, Partovi S, et al. The barrow infundibular PCoA, clipped aneurysm, and reconstructed AChA. (c) 3D reconstruction of (b). (d) Posterior view on clip tips. (e) Medial view with depiction of reconstructed AChA. (f) Same view as (e) without clips

ruptured aneurysm trial: 3-year results. J Neurosurg. 2013;119(1):146-57.

3. Lehecka M, Dashti R, Laakso A, van Popta JS, Romani R, Navratil O, et al. Microneurosurgical management of anterior choroid artery aneurysms. World Neurosurg. 2010;73(5):486-99.

4. Lee Y-S, Park J. Anterior choroidal artery aneurysm surgery: ischemic complications and clinical outcomes revisited. J Korean Neurosurg Soc. 2013;54(2):86-92.

5. Hernesniemi J, Ishii K, Niemela M, Smrcka M, Kivipelto L, Fujiki M, Shen H. Lateral supraorbital approach as an alternative to the classical pterional approach. Acta Neurochir Suppl. 2005;94:17-21.

6. Friedman JA, Pichelmann MA, Piepgras DG, Atkinson JL, Maher CO, Meyer FB, Hansen KK. Ischemic complications of surgery for anterior choroidal artery aneurysms. J Neurosurg. 2001;94(4):565-72. 
Open Access This chapter is licensed under the terms of the Creative Commons Attribution 4.0 International License (http://creativecommons.org/licenses/by/4.0/), which permits use, sharing, adaptation, distribution and reproduction in any medium or format, as long as you give appropriate credit to the original author(s) and the source, provide a link to the Creative Commons license and indicate if changes were made.

The images or other third party material in this chapter are included in the chapter's Creative Commons license, unless indicated otherwise in a credit line to the material. If material is not included in the chapter's Creative Commons license and your intended use is not permitted by statutory regulation or exceeds the permitted use, you will need to obtain permission directly from the copyright holder.

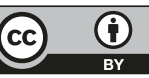

